\title{
Mechanized blood grouping: a hospital trial using an 8-channel grouping machine
}

\author{
G. C. JENKINS, R. G. FEWELL, M. J. LLOYD, JEAN BROWN, J. JUDD¹, \\ R. S. LANE ${ }^{1}$, AND W. J. JENKINS ${ }^{1}$ \\ From the London Hospital, Whitechapel, London and the Regional Blood Transfusion Centre ${ }^{1}$, \\ Brentwood, Essex
}

SYNOPSIS ABO and $\mathrm{Rh}$ (D) groups of 6403 blood samples were assessed on an 8/9-channel autoanalyser in the Serology Department of the London Hospital; the results were independently checked at the Regional Blood Transfusion Centre, Brentwood, using the routine methods for grouping donor blood. Results of this comparative study are given and instances are described in which anomalous results or incorrect groupings occurred; the possible causes are discussed.

The 8/9-channel automated blood group analyser is evaluated in terms of routine hospital laboratory practice.

Samples of blood from a patient population present a far more varied and difficult challenge than those taken from a healthy donor population; therefore, conditions which apply within a hospital laboratory are quite different from those in a regional blood transfusion centre. Most centres in the United Kingdom have adopted automated blood grouping systems and a number of trials have led to improvements in the techniques. Technicon Instruments Ltd have designed an eight-channel blood grouping system which may be used in the transfusion departments of large hospitals.

This report is a comparative account of results obtained from approximately 6400 consecutive blood samples studied by automated techniques at The London Hospital and at the Regional Blood Transfusion Centre in Brentwood. Modifications to the operation of the grouping system introduced during the study are described; suggestions are made for further improvements for its use within a hospital transfusion laboratory.

\section{Materials and Methods}

\section{AUTOMATED BLOOD GROUPING}

\section{Equipment}

The Technicon blood grouping machine under assessment was similar to that described by Scott and Priest (1967). Red cells were treated with bro-

Received for publication 29 April 1975. melin and their agglutination was further enhanced by the use of methyl cellulose (Marsh et al, 1968) and bovine albumin in $1.3 \%$ saline.

Cells were tested with anti-A, anti-B, anti-A + Bo anti-D, and group AB 'inert' serum, while plasma was tested against group A, B, and $O$ red cells: 120 specimens were sampled per hour with a wash sample ratio of $1: 4$.

\section{Collection of Samples}

The machine sampler held $8113 \mathrm{~mm}$ tubes (Labco Ltd). Initially, specimens consisted of $4 \mathrm{ml}$ whole blood mixed with $1 \mathrm{ml}$ of acid-citrate-dextrose (ACD) solution but, after some 3000 samples had been tested, the anticoagulant was changed to $1 \mathrm{mg}$ EDTA per $\mathrm{ml}$ of whole blood and the sample tray was modified by placing a Perspex disc on its underside, thus accommodating conventional $4 \mathrm{ml}$ EDTA sample containers used in routine haematological departments. Avoiding separate sampling for blood grouping greatly facilitated blood collection and EDTA did not influence the results in any way.

\section{Reagents}

The Centre supplied routine antisera having the following titres in saline: anti-A $>1: 120$ against group A, red cells; anti-B $>1: 64$ against group B red cells; anti- $A+B>1: 128$ against group $A_{1}$ red cells and $>1: 64$ against group $B$ red cells. The titres of anti-D sera were above 1:512 against albumin-suspended $R h(D)$ positive red cells.

Anti-A, anti-B, anti-A + B, and group AB inert 


\begin{tabular}{llll}
\hline Antiserum & Dye & $\begin{array}{l}\text { Concentration } \\
\text { of dye in } \\
\text { neat serum }\end{array}$ & Colour \\
\hline Anti-A & Corticol tartrazine S & $0.13 \%(w / v)$ & Yellow \\
Anti-B & Corticol carmosine & $0.08 \%(w / v)$ & Red \\
Anti-A + B & Brilliant blue FCF & $0.08 \%(w / v)$ & Blue \\
Anti-D & Corticol tartrazine S & $0.75 \%(w / v)$ & Green \\
& and brilliant blue FCFO & $0.75 \%(w / v)$ & \\
\hline
\end{tabular}

Table I Dyes and their concentrations used for identification of antisera

sera were diluted $1: 10$ in a solution containing 2 parts $1 \%$ methyl cellulose, 1 part $30 \%$ bovine albumin, and 24 parts $1.3 \%$ saline. Anti-D was diluted $1: 12$ in a solution containing 4 parts $1 \%$ methyl cellulose, 1 part $30 \%$ bovine albumin, and 17 parts $1.3 \%$ saline. It was found convenient to prepare $80 \mathrm{ml}$ of each antiserum at a time since this was sufficient to test 300 specimens.

Each antiserum was identified in the reaction coils and subsequently on the filter paper by colouring the neat serum with a dye (see table I). Preliminary studies at the Centre together with results from tests on more than 100000 donor samples had shown that dyes neither affect serological behaviour nor adversely affect storage of antisera at $-25^{\circ} \mathrm{C}$ (table I).

Reagent red cells collected into ACD were obtained from the Centre. Groups $A_{1} R h(D)$ negative, $B R h(D)$ positive, and $O R h(D)$ positive $\left(R_{1} R_{2}-\mathrm{CDe} / \mathrm{cDE}\right.$, Kell positive) red cells were prepared daily at a concentration of $5 \%$ in a solution containing 2 parts $1 \%$ methyl cellulose, 1 part $30 \%$ bovine albumin, and 26 parts $0.2 \%$ bromelin solution.

Bromelin solution was prepared daily by agitating $2 \mathrm{~g}$ of bromelin powder in $100 \mathrm{ml} 1.3 \%$ saline for $15 \mathrm{~min}$ at room temperature. The solution was $c \in$ ntrifuged and thesupernatant was diluted in 1 litre with $1.3 \%$ saline. The addition of EDTA (dipotassium salt), $2 \mathrm{~g} / \mathrm{l}$, to both the bromelin and wash solutions stabilized the enzyme and retarded fibrin deposition within the machine.

\section{CORRELATION OF RESULTS}

The results of each line output were recorded by a technician at The London Hospital, who added his

\begin{tabular}{llllll}
\hline No. tested & Correct Group & \multicolumn{5}{l}{ Group recorded by Machine } \\
\cline { 3 - 6 } & & $O$ & $A$ & $B$ & $A B$ \\
\hline 2845 & O & 2844 & - & - & 1 \\
2586 & A & - & 2584 & - & 2 \\
699 & B & - & - & 699 & - \\
273 & AB & - & - & 38 & 235 \\
\hline
\end{tabular}

Table II Anomalous $A B O$ cell groups

\begin{tabular}{lllrrr}
\hline No. tested & Correct Group & \multicolumn{5}{l}{ Group recorded by Machine } \\
\cline { 3 - 6 } & & $O$ & $A$ & \multicolumn{1}{c}{ B } & \multicolumn{1}{c}{$A B$} \\
\hline 2845 & O & 2831 & 1 & 2 & 11 \\
2586 & A & - & 2552 & - & 34 \\
699 & B & - & 3 & 693 & 6 \\
273 & AB & - & 7 & 263 \\
\hline
\end{tabular}

Table III Anomalous ABO plasma groups

own interpretation of the blood groups. These results, together with an aliquot of each blood sample, were sent to the Centre for confirmation. At the Centre, the samples were tested by the routine methods used for all blood donor samples. Discrepancies between the laboratories were further investigated at the Centre by conventional manual techniques.

\section{Results}

\section{ABO ANOMALIES}

Of a total of 6403 samples, there were 41 anomalous ABO cell groupings and 64 anomalous plasma groupings (see tables $\mathrm{II}$ and $\mathrm{III}$ ).

One group $O$ cell sample typed as $A B$ with anti$A$, anti-B, and anti-A + B but gave a positive reaction with $A B$ serum: further investigation revealed that the patient had auto-immune haemolytic anaemia (AIHA). Two group A cell samples typed as $\mathrm{AB}$, one due to AIHA (AB serum control positive) the other due to possible misreading of the cell group since a weak result was recorded with anti-B. Thirty-eight group AB cell samples grouped as $B$ in the cell group (negative with anti-A) and these were subsequently grouped as $\mathbf{A}_{2} \mathbf{B}$.

Fifty-one plasma groups were incorrectly grouped as $A B$, presumably due either to failure of the machine to sample correctly or to weak anti-A/anti$B$ in the patient's plasma. Ten group AB plasma samples gave anomalous results: seven grouped as B, attributed to the presence of anti-A; three grouped as A, due to misreading or to the presence of irregular antibodies.

Eight samples were grouped incorrectly by both cell and plasma tests. Five group $\mathbf{A}_{2} B$ samples with anti $\mathbf{A}_{1}$ in the plasma were grouped as $\mathbf{B}$. One group $A$ sample was grouped as $A B$, giving an apparent weak result with anti-B in the cell group together with a negative result in the plasma against $B$ cells. Subsequent re-examination of the results showed that no reaction was obtained with anti-B serum. Two group AB samples were grouped as $\mathbf{A}$ due to weak reactions with anti-B in the cell group and misreading in the plasma group. 


\begin{tabular}{lllc}
\hline No. tested & Correct Group & \multicolumn{2}{c}{ Group recorded by Machine } \\
\cline { 3 - 4 } & & D positive & D negative \\
\hline 5356 & D positive & 5350 & 6 \\
1016 & D negative & 6 & 1010 \\
31 & $D^{u}$ & 3 & 28 \\
\hline
\end{tabular}

Table IV $R h(D)$ grouping

RH(D)-GROUPING

Analysis of the $R h(D)$-grouping results is shown in table IV. Of 6403 samples, 1010 were grouped correctly as D-negative and 5350 correctly as $D$-positive. Of 31 samples later identified as $\mathrm{D}^{\mathrm{u}}, \mathbf{2 8}$ grouped as D-negative and 3 grouped D-positive; 6 D-positive samples were also grouped as D-negative.

Six D-negative samples were grouped as Dpositive; one of these was from a case of AIHA (AB serum control positive), and subsequent examination of the other five results showed them to be Dnegative; the results had been either read or transcribed incorrectly.

\section{Discussion}

The purpose of this study was to examine the performance and test the reliability of the Technicon eight-channel analyser in hospital blood bank practice. Results showed that particular attention must be given to the choice of reagents used in the instrument, to the reading, transcription, and interpretation of reactions in order to achieve the high degree of accuracy demanded of blood grouping procedures.

Of all $\mathrm{ABO}$ groups, $1.6 \%$ resulted in easily recognizable anomalies between cell and plasma tests requiring further investigation by manual techniques. This is not a great inconvenience, but it could cause a dangerous delay in the supply of compatible blood for urgent transfusion. A more serious problem arose when wrong $\mathrm{ABO}$ groups were not associated with evident anomalies, for example, eight group $\mathbf{A}_{2} \mathbf{B}$ patients, of whom five had anti-A $\mathbf{A}_{1}$ in their plasma and thus were incorrectly grouped. In this situation, if a nine-channel analyser is used, group $\mathrm{A}_{2}$ cells may be included, thus aiding the interpretation of results.
While it is disappointing that $90 \%$ of $\mathrm{D}^{\mathrm{u}}$ samples were incorrectly grouped as D-negative and that six D-positive samples were wrongly grouped as Dnegative, it is extremely serious to note that five $D$ negative samples were grouped as D-positive in spite of the correct behaviour of the machine. These five samples were incorrectly read on the filter paper, underlining the need for rigid control of the interpretation and transcription of results. Loading of the is sample tray should be checked by an independent $\overrightarrow{0}$ worker before the testing of samples, and reactions on the filter paper should be read by the instrument operator and by an independent worker. The protocols and their interpretation should then be checked against the filter paper.

The reagents supplied by the Centre were the same as those used regularly in routine grouping of blood donor samples in a 15-channel analyser. The anti-A, 8 anti-B, and high-titre group $O$ sera were obtained $ᄋ$ from unimmunized donors but selected on the basis of their avidity and titre against appropriate cells. Within the transfusion service, when selecting donors, it is known that, using such reagents, reactions with weak group $A$ and $A B$ sub-types are often below the sensitivity of machines, and precautions include the grouping of all apparent groug 6 B samples by a more sensitive manual technique or It is suggested that similar precautions should taken in hospital laboratories.

The authors are conscious of the danger of contamination of the filter paper by hepatitis B antigen. Technicians handling the paper should be aware of this hazard and should use plastic gloves, finally disposing of the paper by incineration.

The authors thank Miss Margaret Kenwright of The London Hospital, and the technicians at the Regional Blood Transfusion Centre for their ? valuable assistance during this investigation.

\section{References}

Marsh, W. L., Nichols, M., and Jenkins, W. J. (1968). Automated detection of blood group antibodies. J. med. D Lab. Technol., 25, 335-342.

Scott, G. L. and Priest, C. J. (1967). Experiences with automatic equipment for routine blood-group serology. Brit. $N$ J. Haemat., 13, Suppl., 40-49. 